\title{
Linfangioleiomiomatosis pulmonar, una rara enfermedad pulmonar: presentación de un caso clínico
}

\author{
JOSÉ L. PINEDA-BOCANEGRA*, KATYA LISSETTE VELÁSQUEZ-CANTILLO*, \\ RONALD MAESTRE-SERRANO** y ERNESTO AGUSTÍN SANTIAGO-HENRÍQUEZ***
}

\section{Pulmonary lymphangioleiomyomatosis, a rare lung disease: presentation of a clinical case}

Introduction: Pulmonary lymphangioleiomyomatosis (LAM) is a rare and progressive disease; characterized by airway, lymphatic and blood vessels-smooth muscle cells excessive proliferation. Added to the abnormal cell growth, parenchymal cystic degeneration is present, which can be reflected initially as a asymptomatic course and can progress to severe gaseous exchange deterioration and fulminating respiratory insufficiency. Case description: A 41-year-old female patient with a clinical course consisting of occasional dry cough, associated with mild pleuritic pain on the right side of thorax. As no improvement was achieved, thoracic imaging study was performed, where a right pneumothorax was found. Tomography images showed multiple lung cystic lesions. Anatomopathological study reports structural changes compatible with LAM. Conclusion: Given the simplicity of the symptoms that LAM can debut with, its diagnostic confirmation is generated in advanced stages of the disease, when the important pulmonary damage leads to the appearance of clinical factors with greater impact on the general state of patients so early thoracic imaging studies gain vital importance.

Key words: Lymphangioleiomyomatosis; pneumothorax; tomography, X-Ray computed; lung; myocytes, smooth muscle.

\section{Resumen}

Introducción: La linfangioleiomiomatosis Pulmonar (LAM) es una rara y progresiva enfermedad; caracterizada por proliferación excesiva de células musculares lisas a partir de vasos linfáticos, sanguíneos y vías aéreas. En conjunto al anormal crecimiento celular descrito, se aprecia degeneración quística difusa del parénquima pulmonar, lo que puede reflejarse desde cuadros completamente asintomáticos hasta el deterioro severo del intercambio gaseoso con insuficiencia respiratoria fulminante. Descripción del caso: Paciente femenino de 41 años de edad, con cuadro clínico consistente en tos seca ocasional, asociada a dolor leve de características pleuriticas en 'puntada de costado'derecha. Ante la no mejoría clínica, se indica estudio imagenológico donde se demuestra neumotórax espontáneo derecho. En estudio tomográfico se aprecian además lesiones pulmonares quísticas. El estudio anátomo-patológico demuestra cambios estructurales que se reportan compatibles con LAM. Conclusión: Dada la simplicidad de los síntomas con que la LAM puede debutar, su confirmación diagnóstica se genera en fases avanzadas de la enfermedad, cuando el daño pulmonar importante conlleva a la aparición de factores clínicos con mayor repercusión sobre el estado general de los pacientes por lo que la realización de estudios imagenológicos tempranos gana vital importancia.

Palabras clave: Linfangioleiomiomatosis; neumotórax; tomogrrafia axial computada; pulmón; miocitos, músculo liso.

* Residente tercer año Medicina Interna. Universidad Libre Seccional Barranquilla, Facultad de Ciencias de la Salud. Barranquilla, Colombia.

** Biólogo, PhD. Docente-investigador. Grupo de Investigación Salud Pública UNILIBRE (GISPUL), Universidad Libre Seccional Barranquilla, Facultad de Ciencias de la Salud. Barranquilla, Colombia.

*** Médico, Internista-Neumólogo. Servicio de Neumología Clínica General del Norte. Barranquilla, Colombia; Docente, Universidad Libre Seccional Barranquilla, Facultad de Ciencias de la Salud. Barranquilla, Colombia. 


\section{Introducción}

La linfangioleiomiomatosis pulmonar (LAM) es una rara y progresiva enfermedad, que se caracteriza por la proliferación anormal de células musculares lisas a partir de vías aéreas, vasos linfáticos y sanguíneos. De forma paralela se desarrolla remodelación quística difusa del parénquima ${ }^{1,2}$. Se conocen dos formas clínicas de LAM: Una variante esporádica y otra asociada a esclerosis tuberosa (ET). Existen reportes de casos en EE.UU., Europa y Asia, a partir de los cuales se han generados datos epidemiológicos de la enfermedad ${ }^{3}$. LAM afecta a mujeres, con una tasa de prevalencia mundial estimada de 2,6 casos por cada 1.000 .000 de mujeres ${ }^{3-5}$. Según la Federación Colombiana de Enfermedades Huérfanas (FECOER), existen 20 pacientes identificadas con esta enfermedad a partir de la recopilación de casos publicados ${ }^{4}$. La edad promedio en el momento del diagnóstico es de 35 años, aunque la enfermedad ha sido descrita en niñas y ancianas ${ }^{6,7}$. LAM ha sido denominada la gran simuladora, ya que presenta gran variedad de manifestaciones clínicas y se asemeja a enfermedades pulmonares comunes como el asma, la enfermedad obstructiva pulmonar entre otras. El síntoma inicial en la mayoría de los pacientes es la disnea secundaria a neumótorax espontáneo y con menor frecuencia el dolor torácico, tos y hemoptisis ${ }^{7}$. Las lesiones histopatológicas generadas por la LAM pueden extenderse a regiones extra torácicas, generando ocasionalmente manifestaciones extrapulmonares como sangrado a partir de angiomiolipomas abdominales y pélvicos que podrían preceder por años la aparición de síntomas respiratorios ${ }^{8,9}$.

Los exámenes de rutina pueden ser de apoyo pero no de diagnóstico. La radiografía de tórax puede no revelar cambios patológicos en las primeras fases de la enfermedad, o presentar neumotórax espontáneo, derrame pleural, infiltrados retículonodulares, quistes o bulas en etapa más tardía. La evaluación espirométrica puede ser normal o reflejar patrones restrictivo u obstrucción, siendo el último el más reportado hasta en un tercio de los casos, sin mejoría tras la administración de un $\beta 2$-agonista adrenérgico ${ }^{8}$.

La tomografía axial computada (TAC), puede revelar múltiples lesiones de aspecto quístico con paredes delgadas que exhiben distribución difusa e intercalan con áreas de parénquima imagenológicamente normal. La extensión del estudio tomográfico a la región abdomino-pélvica permite valorar la presencia de masas correspondientes a los clásicos angiomiolipomas ${ }^{5,9}$.
El 'estándar de oro' para el diagnóstico de LAM es la biopsia de tejido pulmonar comprometido, que mostrará infiltración nodular por células musculares lisas anormales, denominadas células LAM. Estos hallazgos se complementan con tinciones especiales (Tricromo) y marcación mediante inmunohistoquímica (AML). En pacientes que no pueden ser sometidos a biopsia pulmonar debido al deterioro importante de su clase funcional, los ganglios y angiomiolipomas abdominales, pueden participar como fuente de muestreo histopatológico ${ }^{10}$.

\section{Presentación de caso clínico}

Paciente de 41 años de edad, de género femenino; docente de profesión y sin antecedentes patológicos, ni toxicológicos importantes. Manifestó a su ingreso, cuadro clínico de una semana de evolución consistente en disnea de medianos esfuerzos, que se acompañó en los dos últimos días de tos seca ocasional sin predominio de horario, con dolor leve de características pleuríticas en 'puntada de costado' derecha; sintomatología que no mejoró con la ingesta de antigripales, ni analgésicos convencionales, motivo por el cual decidió consultar. La paciente ingresó en buenas condiciones generales, eupneica, tolerando oxigeno ambiente con buen patrón ventilatorio, pero con disminución de ruidos respiratorios en hemitórax derecho a la auscultación. En la radiografía de tórax, se evidenció patrón reticular bilateral difuso y neumotórax derecho mayor de un 50\% (Figura 1), por lo que se realizó toracotomía cerrada derecha, obteniendo la reexpansión pulmonar parcial con posterior resolución total del mismo. La tomografía axial computada (TAC) de tórax evidenció además numerosas lesiones quísticas pulmonares (Figura 2a, 2b). Dentro de los posibles diagnósticos de enfermedad pulmonar bulosa se descartó el déficit de $\alpha 1$-antitripsina, encontrándose su nivel sérico por encima de los niveles de referencia y se planteó la LAM como principal sospecha diagnóstica. Posterior a la realización de la videotoracoscopia (VATS), lobectomía superior derecha, decorticación y pleurectomía parcial, el estudio histopatológico reveló proliferación de fibras de músculo liso que se tiñen con tricromo y positividad para el marcador LAM (células LAM) compatible con la enfermedad.

Dentro de los estudios complementarios realizados, se encuentran ecografía renal y vías urinarias, tomografía axial computarizada abdominal, además de TAC cerebral simple, en donde no se 


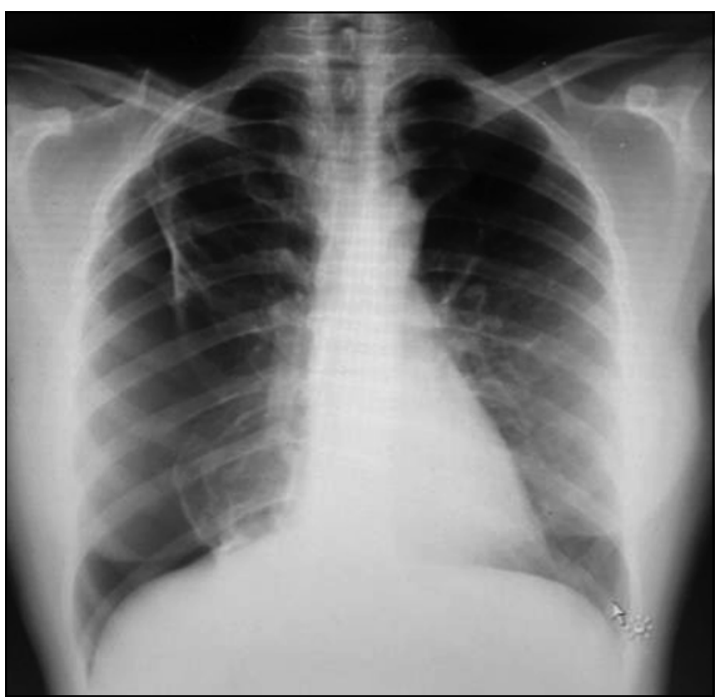

Figura 1. Radiografía en proyección póstero-anterior muestra neumotórax derecho $>50 \%$ y un patrón reticular pulmonar. halló compromiso de sistema nervioso central, ni abdominal. La paciente completó dos años de tratamiento con sirolimus $2 \mathrm{mg}$ cada $24 \mathrm{~h}$; el cual es un inhibidor de la vía de señalización mTOR (mammalian target of rapamycin), critica para la diferenciación y proliferación celular; con respuesta favorable sin deterioro agregado de la función pulmonar y totalmente asintomática. Las pruebas de función pulmonar realizadas antes y posterior (14 meses después) al tratamiento con sirolimus, han mostrado que la función pulmonar ha disminuido lentamente (Tabla 1).

\section{Discusión}

La linfangioleiomiomatosis pulmonar fue descrita y conocida en la literatura histopatológica de la época como "cirrosis muscular pulmonar". Los primeros informes provenían de casos de mujeres
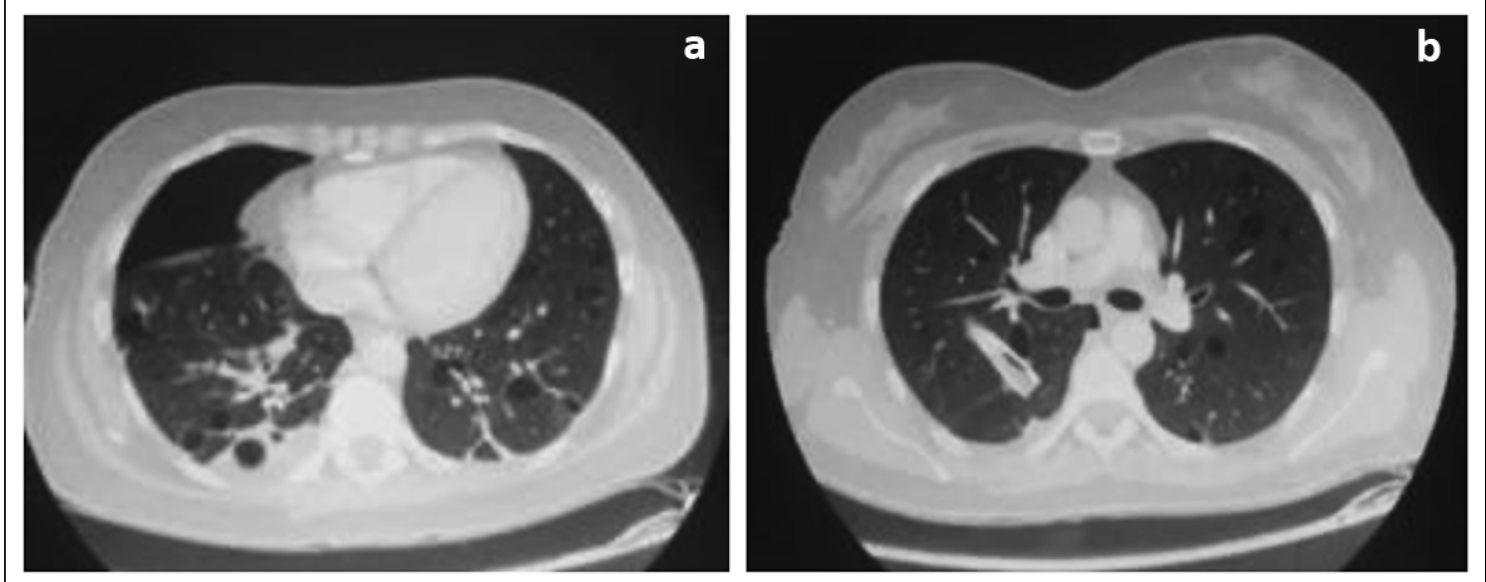

Figura 2. Tomografía axial computada de tórax: (a) Evidencia neumotórax además de múltiples bulas y compromiso parenquimatoso; (b) Lesiones quísticas, de pared fina, comprometiendo el parénquima pulmonar con reexpansión pulmonar con la colocación de tubo de toracotomía.

Tabla 1. Resultados de la espirometría antes y después de tratamiento con sirolimus en la paciente objeto de estudio

\begin{tabular}{|ccc|}
\hline Espirometría & Antes del tratamiento & 14 meses después \\
$\mathrm{CVF}$ & 64 & 59 \\
$\mathrm{VEF}_{1}$ & 64 & 59 \\
$\mathrm{VEF}_{1} / \mathrm{CVF}$ & 99 & 99 \\
\hline
\end{tabular}

CVF: Capacidad vital forzada; $\mathrm{VEF}_{1}$ : Volumen espiratorio forzado en el primer segundo. Valores expresados en $\%$ del valor teórico. 
que desarrollaban insuficiencia respiratoria terminal y cuyos estudios patológicos postmorten describían cambios quísticos y extensa proliferación de tejido muscular liso. Se presenta normalmente con disnea progresiva, neumotórax espontáneo recidivante $\mathrm{y}$ en ocasiones con hemoptisis ${ }^{4,10}$. No se ha descrito un tratamiento curativo de la enfermedad. Existen reportes de supervivencias entre el $38 \%$ y $78 \%$ a los 8,5 años de los casos. Esta enfermedad se puede presentar en asociación con otras condiciones, por ejemplo en complejo con esclerosis tuberosa ${ }^{3}$. La presentación clínica de la paciente correspondía a un cuadro insidioso indiferenciado hasta la aparición del neumotórax espontáneo. Esto sumado a la presencia de lesiones parenquimatosas típicas; y con niveles elevados de $\alpha 1$ anti-tripsina; conllevó a la sospecha diagnóstica confirmada mediante biopsia de tejido pulmonar ${ }^{11}$.

Se han desarrollado estudios con sirolimus, que han demostrado el efecto de este tratamiento sobre la disminución de la progresión del daño funcional pulmonar ${ }^{12,13}$. Esto se ha observado en la paciente analizada en este caso clínico, pero de forma lenta y no a lo esperado en los pacientes que no reciben el tratamiento. Para concluir acerca del efecto del tratamiento con sirolimus en la paciente analizada, se recomienda en el tiempo realizar más controles tanto imagenologicos como de función pulmonar.

\section{Conclusión}

La presencia de neumotórax espontáneo en una mujer en edad reproductiva, debe generar sospechas acerca de la posibilidad de LAM. Si la TAC pulmonar muestra el patrón quístico característico, asociado a elevación del 'factor de crecimiento endotelial vascular D' nos confirmaría diagnóstico de LAM, en caso de tener dudas con el patrón imagenologico o no obtener la elevación del VEFG (vascular endothelial growth factor) estaría justificado obtener una biopsia pulmonar para así obtener el diagnóstico, dado el pronóstico de la enfermedad. Para el personal médico la LAM plantea un desafío, tanto en su diagnóstico, como en el manejo, debido a su patrón restrictivo, las complicaciones por los episodios de neumotórax, la hemoptisis y los cuadros de inestabilidad hemodinámica

El tratamiento para esta enfermedad depende de la función y compromiso pulmonar; por lo tanto, se recomienda trasplante pulmonar si esta se encuentra en estado avanzado o etapa terminal.

\section{Aspectos éticos}

Esta comunicación se acoge a las recomendaciones éticas de la declaración de Helsinki, relacionadas a las investigaciones en seres humanos. Su publicación obtuvo el consentimiento por parte de la paciente objeto de estudio y fue aprobado por el comité de ética de la Clínica General del Norte de la ciudad de Barranquilla, Colombia.

\section{Bibliografía}

1.- JOHNSON SR. Lymphangioleiomyomatosis. Eur Respir J 2006; 27: 1056-65.

2.- MCCORMACK FX. Lymphangioleiomyomatosis: a clinical update. Chest 2008;133: 507-16.

3.- MATSUI K, TATSUGUCHI A, VALENCIA J, YU ZX, BECHTLE J, BEASLEY MB, et al. Extrapulmonary lymphangioleiomyomatosis (LAM): clinicopathologic features in 22 cases. Hum Pathol 2000; 31: 1242-48.

4.- ANTÓN E, CASANOVA A, XAUBET A, ROMÁN A, VILLENA V, MONTERO MC. Lymphangioleiomyomatosis: a study of 72 patients from the Spanish register. Sarcoidosis Vasc Diffuse Lung Dis 2009; 26: 85-91.

5.- FECOER. Federación Colombiana de Enfermedades Raras. ¿Puedes decir Linfangioleioangiomatosis? Disponible en: http://www.fecoer.org/puedes-decirlinfangioleiomiomatosis

6.- ROMAN A, ARISTIZÁBAL D, PALLISA E, MAJÓ J, ISCAR M, MONFORTE V, et al. Linfangioleiomiomatosis: estudio de 15 pacientes. Med Clin (Barc) 2000; 115: 98-102.

7.- HO TB, HULL JH, HUGHES NC. An 86-year-old female with lymphangioleiomyomatosis. Eur Respir J 2006; $28: 1065$.

8. JOHNSON SR, TATTERSFIELD AE. Clinical experience of lymphangioleiomyomatosis in the UK. Thorax 2000; 55: 1052-57.

9.- KITAICHI M, NISHIMURA K, ITOH H, IZUMI T. Pulmonary lymphangioleiomyomatosis: a report of 46 patients including a clinicopathologic study of prognostic factors. Am J Respir Crit Care Med 1995; 151: 527-33.

10.- TANAKA H, IMADA A, MORIKAWA T, SHIBUSA T, SATOH M, SEKINE K, et al. Diagnosis of pulmonary lymphangioleiomyomatosis by HMB45 in surgically treated spontaneous pneumothorax. Eur Respir J 1995; 8: 1879-82.

11.- TAVEIRA-DASILVA AM, MOSS J. Clinical features, epidemiology a therapy of limphangioleiomyomatosis. Clinical Epidemiology 2015; 7: 249-57.

12.- HAYASHIDA M, SEYAMA K, INOUE Y, FUJIMOTO $\mathrm{K}, \mathrm{KUBO} \mathrm{K}$. The epidemiology of lymphangioleiomyo- 
matosis in Japan: A nationwide cross-sectional study of presenting features and prognostic factors. Respiratory 2007; 12: 523-30.

13.- MCCORMACK F, INOUE Y, MOSS J, SINGER L,
STRANGE CH, NAKATA K, et al. Multicenter International Lymphangioleiomyomatosis Efficacy of Sirolimus Trial. Am J Respir Crit Care Med 2011; 183 : A6417.

Correspondencia a:

Ronald Yesid Maestre Serrano, Ph D

Universidad Libre Seccional Barranquilla,

Facultad de Ciencias de la Salud. Km 7 Antigua vía

Puerto Colombia. AA 1752, Barranquilla. Colombia.

Email: rmaestre@unilibrebaq.edu.co ronaldy.maestres@unilibre.edu.co 